\title{
ON DYNAMIC ANALYSIS OF CONTACT PROBLEMS WITH FREEFORM SURFACES: A KNEE JOINT 3D-STUDY
}

\author{
Margarida Machado (1), Paulo Flores (1), Daniel S. Lopes (2), Jorge Ambrósio (2) \\ 1. Centre for Mechanical and Materials Technologies, University of Minho, Portugal \\ 2. IDMEC/Instituto Superior Técnico, Technical University of Lisbon, Portugal
}

\section{Introduction}

One of the biomechanical roles of the knee joint is to withstand high loading forces. Thus, this articulation is quite susceptible to injuries and diseases, which may cause anomalous contact loads, asymmetrical gait patterns and local pain that, ultimately, leads to a knee replacement. In view of that, identifying and quantifying the loads placed on the human knee is critical for understanding realistic joint mechanics. Since there is no standard non-invasive experimental approach able to measure in vivo knee dynamic loads, such quantities have to be predicted by making use of computational methods [Bei et al., 2004, Machado et al., 2010]. The efficiency of these computational methods is a primary concern for a contact formulation to be used in multibody system (MBS) dynamics. In fact, it has been recognized by many researchers that most of the time consumed in simulating contact phenomena is spent on the contact detection phase [Choi et al., 2010]. This computational time motivated the development of an efficient methodology to predict the contact forces that is here presented.

\section{Contact Methodology}

The proposed contact methodology deals with three main features: (i) generation and representation of the contact surfaces; (ii) determination of the contact detection between potential contact points; (iii) evaluation of the contact forces.

The geometric description of the knee contacting surfaces are generated and represented by using parametric functions, such as NURBS surfaces, due to its simplicity and easiness to handle freeform surfaces [Bei et al., 2004]. This task is carried out in a pre-processing unit performed preliminarily to the implementation of the MBS formulation. Essential to the success of the proposed methodology is the accurate prediction of the location of the contact points [Machado et al., 2010]. For this purpose, the relative distance between the candidate contact points are computed and utilized with a user-specific tolerance to determine if the bodies are in contact or not. The actual contact points are selected as those that correspond to the maximum relative penetration. In order to compute the knee contact forces, an appropriate constitutive law based on the Hertzian theory augmented with a dissipative term is utilized [Machado et al., 2010]. Then, the intra-joint contact forces are incorporated into the equations of motion that govern the dynamics of the system. Several computational simulations are performed using a 3D-model of the human knee joint, which is composed by two bodies, femur and tibia, and eight nonlinear springs that represent the knee ligaments.

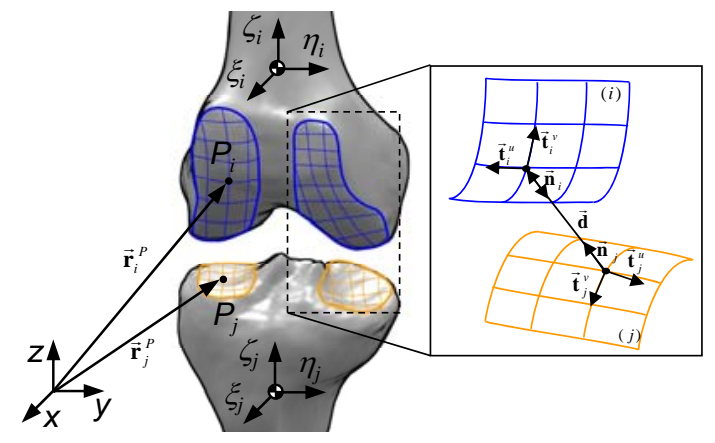

Figure 1: MBS knee model with contact surfaces.

\section{Conclusions}

A contact methodology, developed under the framework of MBS dynamics, is utilized to model the human knee and to predict the dynamic loads at this joint. It is worth noting that the general structure of the proposed contact code enables it to be used to analyze other human joints or even artificial joints. Moreover, the pre-processing unit included to generate and represent the freeform contact surfaces demonstrated to be efficient and suitable for biomechanical simulations, which have real-time computation demands.

\section{Acknowledgments}

The authors would like to thank the support of Fundação para a Ciência e a Tecnologia (FCT), under the projects DACHOR (MIT-Pt/BSHHMS/ 0042/2008) and BIOJOINTS (PTDC/EME-PME/ 099764/2008). The first and third authors thank also FCT for the PhD grants (SFRH/BD/40164/ 2007) and (SFRH/BD/47750/2008).

\section{References}

Bei et al., Med Eng Phys, 26: 777-789, 2004.

Choi et al., Multibody Syst Dyn, 23: 99-120, 2010. Machado et al., Nonlinear Dyn, 60: 459-478, 2010. 